\title{
Research on Prediction of Shelf Life of Cookie Comprehensive Index Based on Bp Neural Network
}

\author{
Juxian $\mathrm{Wu}^{1}$, Baiqi Huang ${ }^{1}$, Xuexun Cheng ${ }^{1}$, Chuhuan Zeng ${ }^{2}$ \\ ${ }^{1}$ Guangdong Polytechnic of Science and Trade, Guangdong, Guangzhou, China \\ ${ }^{2}$ Guangdong Food Industry Association, Guangdong, Guangzhou, China
}

Keywords: Cookie, Shelf life, Prediction, Bp neural network

\begin{abstract}
Cookies are a high-end biscuit product, which is manufactured and exported by a large number of domestic companies. Its accurate shelf life prediction is of great significance to the quality of products and foreign exchange earned from exports. The comprehensive changes of the physical and chemical microorganisms in the sealed environment of the product are closely related to the weight gain rate of the pre-packaged products. This article conducts a comprehensive investigation through the entire pre-packaged cookies. Research on product shelf life and improve efficiency. In the application of the model, the difference between the four predicted values and the actual moisture index is small, $-2.84 \%, 0.5744 \%, 0.5036 \%$, and $0.023 \%$, respectively, which has a good prediction effect.
\end{abstract}

\section{Introduction}

Cookies are a kind of high-end biscuit products, which are produced and exported by a large number of domestic enterprises. When exporting abroad, due to the long-distance transportation of products and the storage environment of imported countries, the shelf life of products is not easy to control, which has long restricted the sales of our baked products abroad. Predicting shelf life has obvious effects on companies to evade foreign technical barriers and improve the quality of export products, as well as to enhance their foreign competitiveness.

Commonly used radar chart evaluation, $\mathrm{Q}_{10}$ equation and Arrhenius equation shelf life prediction use, due to constant temperature and humidity experimental equipment, packaging material aging accelerated experimental equipment and other aspects, technical personnel level, etc., to promote the technical personnel of small and medium-sized enterprises Difficult, it is more practical to explore a simpler, effective and easy to promote shelf life prediction program for SMEs.

Artificial neural network (ANN) referred to as neural network, proposed by Rumel Hant and McClelland in 1986, is currently one of the most widely used and most achieved neural networks. There are more than 60 commonly used neural networks. A multi-layer feed forward network (abbreviated as BP network) based on the error back propagation algorithm (Back Network) can simulate the interaction between the real world of biological nervous system and objects.

Artificial neural network processing information is to train the neural network through information samples, so that it has the memory and recognition ability of the human brain, and completes the famous information processing function. It does not need any a priori formula, it can automatically summarize the rules from the existing data, and obtain the internal laws of these data. The computing system formed by the units to be processed is connected to each other according to a certain logic. It has good self-learning and self-learning.

The ability to adapt, parallel process and transform non-linear shapes. If the signal output by the output layer does not match the expected output, then go to the next process, which is the back propagation of the error. The advantage is the modeling of solving nonlinear problems, with strong input and output nonlinear mapping capabilities, and it is now widely used in industries such as intelligent manufacturing and data modeling and analysis. Its outstanding advantage is that it has strong nonlinear mapping ability and flexible network structure [1-3]. BP neural network is especially suitable for the problem of non-deterministic reasoning, judgment, recognition and 
classification with complicated causality. For any set of random, normal data, artificial neural network algorithm can be used for statistical analysis to make fitting and prediction.

The research uses pre-packaged cookie sample detection, uses the moisture index value as the input of the BP neural network, and the actual shelf life as the output of the BP neural network, sets the hidden layer nodes, and trains to construct the best BP neural network model. This model is used to obtain the actual shelf life of cookies, which provides a quick detection method for baking companies to obtain the shelf life of cookie products, and also provides a certain theoretical basis for the research on the shelf life prediction of baked products.

\section{Based on Bp Neural Network Algorithm, Establish a Mining Model}

\subsection{Sample Extraction}

There are several basic principles for sample extraction. First, variables that greatly affect the output of the result and are easily detected or extracted are used as input variables. Second, try not to have too much correlation or non-correlation between input and output. At the same time, they are divided into two categories according to the nature of input and output: character variables and numeric variables. The latter can be divided into discrete variables and continuous variables. Character variables can only be processed by the network when converted to discrete variables. Third, it is necessary to use signal processing or feature extraction techniques in the original data to extract parameters that can reflect the characteristics of the network as input, that is, to perform normalization processing.

\subsubsection{Sample Evaluation}

The BP algorithm uses "training" to evaluate the input and output of this link, and the appropriate linear or nonlinear relationship. The process of "training” evaluation can be divided into two stages: forward transmission and backward feedback:

Forward transmission phase:

(1)Take a sample from the sample set $P_{i}, Q_{j}$, Enter $P_{i}$ into the network;

(2)Calculate error measure $E_{1}$ and actual output $O_{i}=F_{L}\left(\ldots\left(F_{2}\left(F_{1}\left(P_{i} W^{(1)}\right) W^{(2)}\right) \ldots\right) W^{(L)}\right)$;

(3) Make adjustments $W^{(1)}, W^{(2)}, \ldots W^{L}$ to the weight values,once and repeat this cycle,until $\sum E_{i}<\varepsilon$ backward feedback stage (error propagation stage);

(1)Calculate the difference between actual output $O_{p}$ and ideal output ${ }^{Q_{i}}$;

(2)Adjust output layer weight matrix with output layer error;

(3) $E_{i}=\frac{1}{2} \sum_{j=1}^{m}\left(Q_{i j}-O_{i j}\right)^{2}$;

(4) Use this error to estimate the error of the direct leading layer of the output layer, and then use the error of the output layer leading layer to estimate the error of the previous layer. In this way, the error estimates of all other layers are obtained;

(5) And use these estimates to modify the weight matrix. Form a process that passes the error shown at the output to the output in stages in the opposite direction to the output signal.

The error measure of the network about the entire sample set:

$$
E=\sum_{i} E_{i}
$$

The length of the training time is closely related to the initialization method, so the initialization of the network weights is used to determine where the error starts. Due to the symmetry of the neuron action function relative to the origin, the net input and output of each node near the zero point is at the midpoint of the action function, and the change in this position is the most sensitive 
and far from saturation. Action function area. Therefore, it is necessary to accelerate the learning of the network by choosing a smaller initial weight and making the initial weights +1 and -1 equal.

As shown in Figure 1.

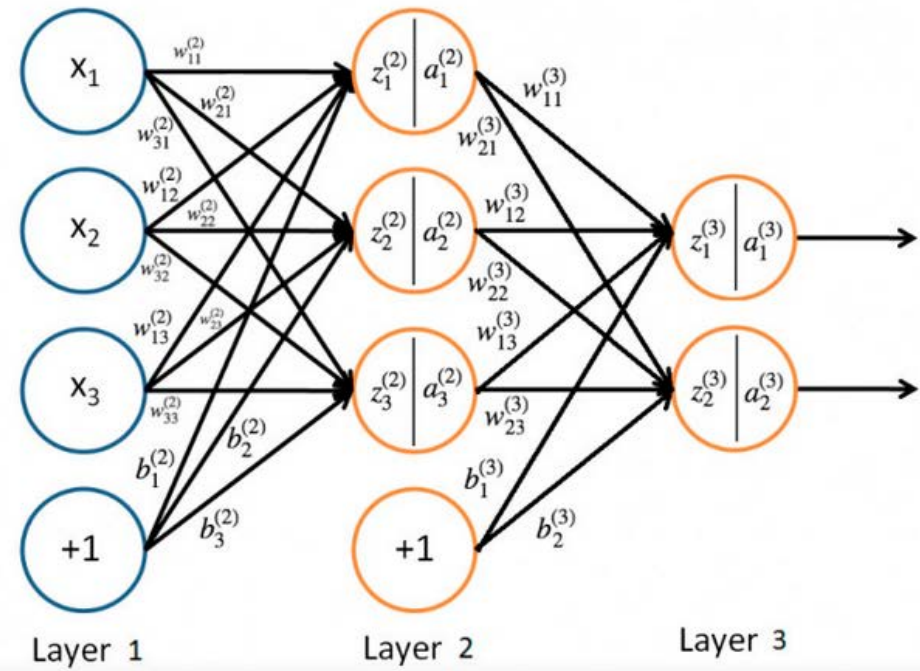

Fig.1 Bp Network Model

\subsubsection{Experimental Method}

Equipment:

BS124 electronic balance, biscuit moisture detector, mortar.

Experimental method:

Using the packaging materials used by the company's current production products, each $10 \mathrm{~g}$ pre-packaged sample was prepared and placed in an acceleration box at a constant temperature and humidity environment of $25{ }^{\circ} \mathrm{C}$ for experiments. The data of the weight gain rate was measured every 45 days, and the increase was more than $0.016 \%$. The empirical data of the weight rate is limited, and the moisture index exceeds the empirical value. The complete cycle is the shelf life of biscuits in December, and the moisture index is measured at the end of the shelf life.

\section{Results and Analysis}

\subsection{Bp Neural Network Anti-Truth Experiment}

On the basis of cookie retention sample test data, based on comparison of relevant literature and historical sample retention observation data of the enterprise [3-11]. The key physical and chemical indicators of the characteristics of the shelf life changes of varieties with different fat content will be different. The key indicators of varieties with high fat are acid value and peroxide value; products with low fat or low initial moisture have a large part of their key indicators It is a moisture index; special varieties such as soft cookies, the key indicators are closely related to moisture and microbial indicators. At the same time, each test requires an unsealed test. The samples after the test cannot continue to be retained for observation. Batch testing can eliminate individual differences, and a large number of retained samples must be compared to improve the accuracy of prediction. Considering the stable internal environment change and the slow penetration of external air in the overall sealed package, it caused physical and chemical microbial reactions inside the package, which comprehensively reflected the overall weight gain. Therefore, consider adopting comprehensive indicators to feed back the change in overall weight gain rate On the shelf life forecast.

In the daily sample retention products of the enterprise, randomly sample quantitative sample data, perform neural network training and simulation on the product moisture index at the end of the sample retention, the target variable is the moisture index after the end of the shelf retention sample, and use the moisture to predict the product data. 
The forecast data is analyzed by BP neural network program in DPS software.

Table 1 Original Test Data

\begin{tabular}{|l|l|l|l|l|l|l|l|l|l|}
\hline No. & \multicolumn{9}{|l}{ Weight gain rate (\%) } \\
\hline 1 & 0 & 0.001642 & 0.003284 & 0.004926 & 0.004926 & 0.011494 & 0.01642 & Missing & 0.0334 \\
\hline 2 & 0 & 0 & 0.001447 & 0.001447 & 0.002894 & 0.007236 & 0.013025 & 0.015919 & 0.0417 \\
\hline 3 & 0 & 0 & 0.002762 & 0.004144 & 0.004144 & 0.006906 & 0.012431 & 0.016575 & 0.0387 \\
\hline 4 & 0 & 0.001437 & 0.00431 & 0.00431 & 0.00431 & 0.008621 & 0.014368 & 0.018678 & 0.0365 \\
\hline 5 & 0 & 0 & 0.002853 & 0.002853 & 0.002853 & 0.008559 & 0.012839 & 0.017118 & 0.0393 \\
\hline 6 & 0 & 0 & 0 & 0.001401 & 0.002801 & 0.005602 & 0.011204 & 0.014006 & 0.0411 \\
\hline 8 & 0 & 0 & 0.00149 & 0.00149 & 0.002981 & 0.005961 & 0.011923 & 0.016393 & 0.0445 \\
\hline 9 & 0 & 0 & 0.001825 & 0.00365 & 0.00365 & 0.009124 & 0.016423 & Missing & 0.0416 \\
\hline 10 & 0 & 0.001479 & 0.001479 & 0.002959 & 0.004438 & 0.007396 & 0.014793 & 0.014793 & 0.0397 \\
\hline 11 & 0 & 0 & 0.001603 & 0.003205 & 0.003205 & 0.008013 & 0.012821 & 0.017628 & 0.0423 \\
\hline
\end{tabular}

Table 2 the Weight Matrix of Each Node in the First Hidden Layer

\begin{tabular}{|l|l|l|l|l|l|l|l|l|}
\hline Matrix data & & & \\
\hline 0.024327 & -0.16168 & 0.374584 & 0.387167 & 0.374065 & -0.34457 & 0.26527 & -0.16354 & 0.370599 \\
\hline-0.56409 & -0.15818 & 0.015012 & -0.35485 & -0.27171 & 0.277746 & 0.558444 & -0.65485 & -0.02756 \\
\hline-0.64379 & -0.12531 & -0.69849 & -0.27913 & -0.37875 & -0.49168 & 0.004624 & 0.16866 & -0.45561 \\
\hline-0.60738 & -0.50886 & -0.23002 & 1.697001 & -0.48123 & -1.83539 & -1.70105 & 1.39733 & -0.88015 \\
\hline 0.139195 & -0.06303 & 0.12277 & -0.67314 & -0.25831 & 0.782814 & 0.304117 & -1.39136 & -0.06399 \\
\hline-0.6624 & -1.10478 & -0.1127 & 1.154757 & -1.0882 & -1.88421 & -1.88787 & 1.718395 & -0.84219 \\
\hline-0.37091 & -0.29678 & -0.32019 & 0.450833 & -0.17878 & -0.33182 & -0.16974 & -0.22992 & 0.274005 \\
\hline 0.593915 & 0.324211 & 0.036936 & -1.29086 & 0.532483 & 1.52067 & 1.028579 & -3.08412 & 0.248892 \\
\hline
\end{tabular}

Table 3 Training Sample Prediction Data

\begin{tabular}{|l|l|l|}
\hline No. & Moisture (\%) & Residual \\
\hline Training samples 1 & 0.033407 & $6.93 \mathrm{E}-06$ \\
\hline Training samples 2 & 0.039297 & $-3.1 \mathrm{E}-06$ \\
\hline Training samples 3 & 0.041101 & $1.04 \mathrm{E}-06$ \\
\hline Training samples 4 & 0.044321 & -0.00018 \\
\hline Training samples 5 & 0.039695 & $-4.9 \mathrm{E}-06$ \\
\hline Training samples 6 & 0.0423 & $-3.5 \mathrm{E}-07$ \\
\hline Mean square error MSE & $5.34 \mathrm{E}-09$ & \\
\hline Residual standard deviation RSE & $8 \mathrm{E}-05$ & \\
\hline Mean absolute deviation MAD & $3.25 \mathrm{E}-05$ & \\
\hline
\end{tabular}

Table 4 Test Sample Prediction Data

\begin{tabular}{|l|l|l|l|}
\hline No. & Moisture (\%) & Error (\%) & Residual \\
\hline Forecast sample 1 & 0.043236 & -2.84 & 0.001536 \\
\hline Forecast sample 2 & 0.039928 & 0.5744 & 0.001228 \\
\hline Forecast sample 3 & 0.042513 & 0.5036 & 0.006013 \\
\hline Forecast sample 4 & 0.033408 & 0.023 & -0.00819 \\
\hline Mean square error MSE & $2.68 \mathrm{E}-05$ & & \\
\hline Residual standard deviation RSE & 0.005975 & \\
\hline Mean absolute deviation MAD & 0.004242 & \\
\hline
\end{tabular}

\subsection{Results Analysis}

Comparing the results obtained by the model with the sample data, the errors of the two groups of experiments are small, and the BP experiment results have good predictability. In the moisture data, 11 samples are divided into 3 varieties, and 3, 3 and 4 sets of parallel samples are made respectively. Among them, the weight gain of sample No. 1 and sample No. 9 exceeded the expected weight gain during the shelf life. After the moisture index test, No. 9 exceeded the national standard level and was judged as unqualified. As a result, the indicator exceeds the standard and is missing, so a set of remaining sample data is added to make a supplementary prediction. The third variety is 4 sets of parallel. The difference between the four predicted values and the actual moisture index is small, respectively $-2.84 \%, 0.5744 \%, 0.5036 \%, 0.023 \%$, and the prediction effect is good. 


\section{Conclusions}

In this experiment, artificial neural network is used to combine cookies with sample data to fully mine the information of the test data to establish a neural network model. The water index data evaluated and predicted by the simulation model, compared with the actual data, the two test errors are small, the BP test results are highly feasible and cost-saving, and reduce energy consumption. It shows that the new model prediction scheme and data processing method proposed by artificial neural network prediction are feasible. This optimized method provides a new way for the design of comprehensive prediction of cookie shelf life.

\section{Acknowledgment}

Fund Project: 2017 Guangdong University Key Research Platform Project Funding (No. 2017GKQNCX031).

\section{References}

[1] Kantardzic, M. Data Mining Concepts, Models, Methods, and Algorithms, Piscataway: IEEE Press, 2002, pp.11-23.

[2] Yu, J.Y., He, X.H. Data Statistical Analysis and SPSS Application, Beijing: People’s Posts and Telecommunications Press, 2003, pp.17-25.

[3] Chen, G., Liu, C.F. Data Mining Algorithm Based on BP Neural Network. Computer and Modernization, no. 10, pp. 20-22, 2006.

[4] Tan, L.Y., Kelly, B., Tassie, P., et al. Research on Data Preprocessing Methods in Data Mining, Hefei: China University of Science and Technology Press, 2004, pp.97-102.

[5] Mei, M.X., Liu, W., Song, Y., et al. Prediction Model of Storage Shelf Life of Low-Salt Pickled White Radish in Different Packages. Food Fermentation Industry, vol. 43, no. 4, pp. 69-77, 2017.

[6] IFT. Shelf Life of Foods. Journal of Food Science, no. 39, pp. 1-4,1974.

[7] Aliee, P. Shelf-life. Nutrition and Food Science, no. 99, pp.131-135, 1999.

[8] IFST. Shelf Life of Foods- Guidelines for its Determination and Prediction, London: IFST, 1993, pp.121-131.

[9] Meginn, C.J. Valuation of Shelf Life. IFST Proceedings, London: IFST, 1982, pp.153- 161.

[10] Dappolonia, B.L., Morad. M.M. Bread staling. Cereal chemistry,1981,58:186- 191.

[11] Roger, D.E., Zeleznak, K.J., Lai, C.et al. Effect of Native Lipids, Shortening, and Bread Moisture on Bread Firming. Cereal Chem, no. 65, pp. 398,1988.

[12] Kada, R., Robinson, M.G., Thibodeau, D.P. et, al. Texture and Other Physic Chemical Properties of Whole Rice Bread. Journal of Food Science, no. 66, pp. 940-944, 2001.

[13] Piazza, L, Masi, P. Moisture Distribution Throughout the Bread Loaf During Staling and its Effect on Mechanical Properties. Cereal Chemistry, no. 73, pp. 320-325, 1995.

[14] Stollman, U., Lundgren, B. Texture Changes in White Bread: Effects of Processing and Storage. Cereal Chemistry, no.164, pp. 230-236,1987. 